\title{
CSCO BC guideline: updates for HER2 positive breast cancer in 2020
}

\author{
Jianbin Li ${ }^{1,2}$, Zefei Jiang ${ }^{1}$ \\ ${ }^{1}$ Department of Breast Oncology, The Fifth Medical Center of Chinese PLA General Hospital, Beijing 100071, China; ${ }^{2}$ Department of Medical \\ Molecular Biology, Beijing Institute of Biotechnology, Academy of Military Medical Sciences, Beijing 100850, China \\ Correspondence to: Zefei Jiang. Department of Breast Oncology, The Fifth Medical Center of Chinese PLA General Hospital, Beijing 100071, China. \\ Email: jiangzefei@csco.org.cn.
}

\begin{abstract}
The Chinese Society of Clinical Oncology (CSCO) guideline for breast cancer (BC) has proved to be some of the most valued clinical guidelines in China. In 2018, the CSCO guideline classified recommendations into three levels. These classifications have been widely accepted and also featured in the CSCO BC guideline published in April this year. HER2 positive is an important subtype of BC. The revolution in treatment patterns for $\mathrm{BC}$ has been accelerated by improvements in both the cost effectiveness and the accessibility of new drugs. This article has been written to offer an interpretation of the CSCO BC guidelines in 2020 .
\end{abstract}

Keywords: Chinese Society of Clinical Oncology guideline for breast cancer; breast cancer (BC); HER2 positive; updates

Received: 28 February 2020; Accepted: 24 March 2020; Published: 10 April 2020.

doi: $10.21037 /$ tbcr.2020.03.05

View this article at: http://dx.doi.org/10.21037/tbcr.2020.03.05

The Chinese Society of Clinical Oncology (CSCO) guideline for breast cancer (BC), which were published in 2017, have been one of the most distinctive sets of clinical guidelines in China (1). HER2 positive is an important subtype, accounting for about $20 \%$ of total BC cases. With the promotion of clinical research, HER2 positive treatments have entered the era of dual-target therapy in the form of trastuzumab and pertuzumab (HP). The approval of new drugs, inclusion of medical insurance, and other factors have also contributed to changes in treatment patterns, especially in recent years. Under these circumstances, the CSCO BC guidelines relating to HER2positive BC have been significantly updated for 2020. This article mainly describes the development and update of the CSCO BC guideline for HER2 positive BC.

\section{Target therapy for metastatic BC}

Although a trastuzumab-based scheme is recommended as the first-line therapy by almost every set of guidelines, because of the influence of neoadjuvant and adjuvant targeted treatment of $\mathrm{BC}$, the lines for anti-HER2 treatment are not clearly defined.

It is important to note that "trastuzumab sensitive" is a more appropriate definition of routine clinical practice when compared with "first line". Since 2018, the reuse of trastuzumab has been put forward for those who have benefitted from neoadjuvant trastuzumab therapy, or have relapsed with at least a 1-year disease free survival after adjuvant trastuzumab treatment, or those who have discontinued their effective trastuzumab in the metastatic stage. Such patients, along with those who have never previously used trastuzumab, may be considered as the trastuzumab-sensitive population and should be recommended a trastuzumab-based scheme in the first instance (Figure 1). Meanwhile, those with progression prior to trastuzumab or those who have relapsed within 1 year after adjuvant trastuzumab should be considered as the trastuzumab-resistant population.

For the trastuzumab-sensitive population, the CLEOPATRA study (2) demonstrated the effect of taxanetrastuzumab-pertuzumab (THP) in metastatic BC. With 


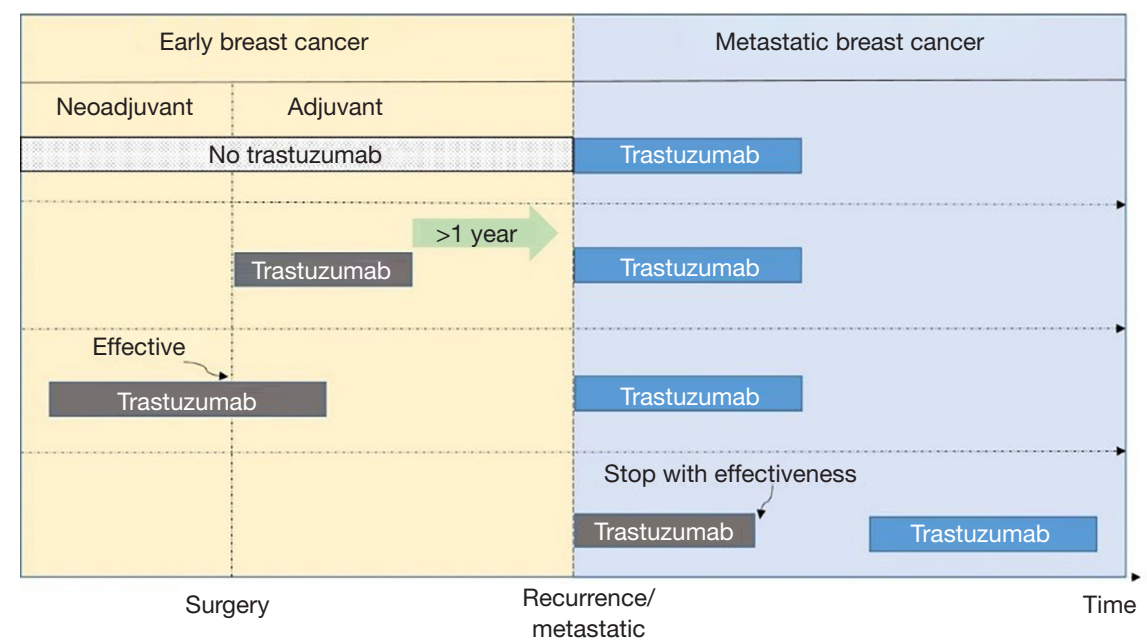

Figure 1 Definition of trastuzumab sensitive population.

Table 1 Recommendation for HER2 positive metastatic breast cancer

\begin{tabular}{llll}
\hline Stratification & Level I recommendation & Level II recommendation & Level III recommendation \\
\hline $\begin{array}{llll}\text { (I) Trastuzumab-naïv; } \\
\text { (II) reuse of trastuzumab }\end{array}$ & $\begin{array}{l}\text { (I) THP (1A); } \\
\text { (II) TXH (1A) }\end{array}$ & $\begin{array}{l}\mathrm{H}+\text { chemotherapy (2A); } \\
\text { including taxane, vinorelbine, } \\
\text { capecitabine }\end{array}$ & $\begin{array}{l}\text { (I) Pyrotinib + capecitabine (2B); } \\
\text { (II) + other chemotherapy (2B) }\end{array}$ \\
Resistance to trastuzumab & Pyrotinib + capecitabine (1A) (I) T-DM1(1A); (II) LX (2B) & $\begin{array}{l}\text { (I) Pyrotinib; (II) TKI + other chemotherapy (2B); } \\
\text { (III) H + other chemotherapy (2B) }\end{array}$ \\
\hline
\end{tabular}

P, pertuzumab; H, trastuzumab; L, lapatinib; T-DM1, ado-trastuzumab emtansine; TKI, tyrosine kinase inhibitor; T, taxane; $\mathrm{X}$, capecitabine.

an increasing amount of evidence and the lowered price of pertuzumab, THP is now a level I recommendation in our guideline (Table 1). However, docetaxel-capecitabinetrastuzumab (TXH) (3) is still an alternative scheme with high cost effectiveness and consensus among Chinese experts, making it a rational level I recommendation option.

One of the concerns that has arisen from the CLEOPATRA study is that only about $20 \%$ of patients had previously received taxanes. Those with prior resistance to taxanes are unlikely to benefit from THP again. For these patients, some clinical studies have verified the feasibility of trastuzumab-pertuzumab combined with other chemotherapy drugs [such as vinorelbine (4)]. Hence, this regimen has been added as level III recommendation in the CSCO BC guideline for 2020.

There have also been updates for those who show resistance to trastuzumab (Table S1). The NCCN guidelines recommend ado-trastuzumab emtansine (T-DM1) as the first-choice treatment and lapatinib as the second for second line therapy. In China, lapatinib combined with capecitabine has already served as the first choice for a long period of time, owing to the unattainability of T-DM1 or any other drugs. Pyrotinib is another oral irreversible tyrosine kinase inhibitor (TKI) for patients with HER2 positive BC. In a phase II clinical study (5), pyrotinib combined with capecitabine has been proved to be an effective and tolerable scheme when compared with lapatinib plus capecitabine. Considering their accessibility, tyrosine kinase inhibitorcontained regimens, pyrotinib, or lapatinib, hold priority over T-DM1 (Figure 2).

The PHENIX trial (6) revealed the promising efficacy of pyrotinib alone for patients with HER2 positive BC who have previously received taxane or trastuzumab. Therefore, pyrotinib alone has been added as a level III recommendation for those with resistance to trastuzumab. As a novel drug developed in China, pyrotinib, whether used alone or in combination, offers another treatment alternative, especially for those with resistance to lapatinib and T-DM1. 


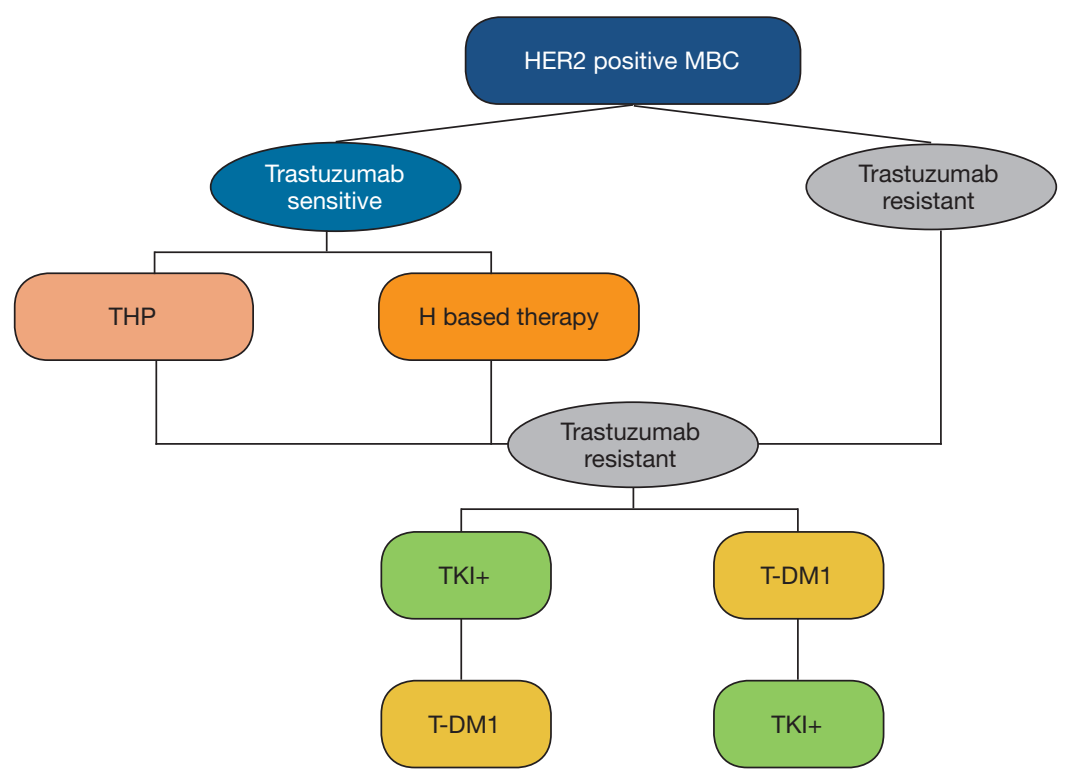

Figure 2 CSCO BC guideline strategies (version 2020): HER2 positive MBC. T, taxane; H, trastuzumab; P, pertuzumab; TKI, tyrosine kinase inhibitor; T-DM1, ado-trastuzumab emtansine; CSCO, Chinese Society of Clinical Oncology; BC, breast cancer.

Table 2 Recommendations for adjuvant target therapy without neoadjuvant therapy

\begin{tabular}{llll}
\hline Stratification & Level I recommendation & Level II recommendation & Level III recommendation \\
\hline Lymph nodes positive & AC-THP (1A); TCbHP (1A) & AC-TH (1A); TCbH (1A) & Neratinib after trastuzumab (2A) \\
$\begin{array}{l}\text { Lymph nodes negative with other risks: } \\
\mathrm{T}>2 \mathrm{~cm} \text {; others (ER negative) }\end{array}$ & AC-TH (1A); TCbH (1A) & AC-THP (2A); TCbHP (2A) & Neratinib after trastuzumab (2B) \\
Lymph nodes negative and T $\leq 2 \mathrm{~cm}$ & $\mathrm{TC}+\mathrm{H}(2 \mathrm{~A})$ & TH (2B) & $\begin{array}{l}\text { Trastuzumab after chemotherapy } \\
\text { (2B) }\end{array}$ \\
$\begin{array}{l}\text { Hormone receptor positive: chemotherapy } \\
\text { free or intolerable to chemotherapy }\end{array}$ & & $\begin{array}{l}\text { Endocrine therapy + } \\
\text { trastuzumab (2A) }\end{array}$ &
\end{tabular}

T, Taxane; P, Pertuzumab; H, Trastuzumab; A, anthracycline; C, cyclophosphamide; Cb, carboplatin.

\section{Adjuvant target therapy without neoadjuvant systemic therapy}

The HERA study (7) confirmed the significance of trastuzumab in HER2 positive adjuvant therapy. However, compared to trastuzumab, domestic experts have rarely regarded dual-targeted therapy as the more appropriate treatment for all population. As neoadjuvant therapy for HER2 positive patients has become more widely applied, the population suitable for initial adjuvant therapy has decreased.

The APHINITY study (8) showed that the addition of pertuzumab to AC-TH (anthracycline plus cyclophosphamide followed by taxane plus trastuzumab) and $\mathrm{TCbH}$ (taxane carboplatin trastuzumab) could improve the invasive disease-free survival of patients, especially for those with positive lymph nodes. On this basis, for patients with positive lymph nodes, both AC-THP and TCbHP have been put forward as level I recommendations, while $\mathrm{AC}-\mathrm{TH}$ and $\mathrm{TCbH}$ schemes have been downgraded to level II (Table 2). For those with risks other than positive lymph nodes (such as tumor lesions $>2 \mathrm{~cm}$ and estrogen receptor negative tumors), AC-TH and TCbH should be the preferred treatment options when cost effectiveness and evidence are taken into account, while AC-THP and TCbHP have been included as level II recommendations.

The ExteNET study (9) has proved that neratinib for 12 months can significantly improve the 2-year invasive disease free survival when received after trastuzumab-based 
Table 3 Recommendations for adjuvant target therapy after neoadjuvant therapy

\begin{tabular}{llll}
\hline Target therapy in neoadjuvant therapy & Stratification & Level I recommendation & Level II recommendation \\
\hline Trastuzumab-only & pCR & Trastuzumab (1A) & HP (2A) \\
Trastuzumab-only & Non-pCR & HP (2A); T-DM1 (1B) & Trastuzumab (2B) \\
HP based therapy & pCR & HP (1A) & Trastuzumab (2B) \\
HP based therapy & Non-pCR & T-DM1 (1B) & HP (2A) \\
\hline
\end{tabular}

$\mathrm{H}$, trastuzumab; P, pertuzumab; T-DM1, ado-trastuzumab emtansine; pCR, pathological complete remission.

adjuvant therapy. Therefore, neratinib is included as the level III recommendation to offer more options to these high-risk patients. However, most of our experts concur that for patients who are receiving trastuzumab, additional pertuzumab should be considered over neranitib.

There is an escalating trend in target treatment for high-risk patients, while de-escalating chemotherapy is also effective for low risk HER2 positive patients in an adjuvant context. For patients without positive lymph nodes and other high-risk factors, de-escalating therapies such as a docetaxel-cyclophosphamide-trastuzumab scheme $(\mathrm{TC}+\mathrm{H})$ and a weekly trastuzumab plus paclitaxel scheme (TH) are recommended in the CSCO BC guideline. For hormone receptor positive patients whose risk of recurrence is too low to receive chemotherapy or those who exhibit intolerance to chemotherapy, endocrine therapy plus trastuzumab is also included as a level II recommendation in the CSCO BC guideline. The evidence level of endocrine therapy plus trastuzumab is $2 \mathrm{~A}$, which is attributed to the lack of necessary data but high consistency among domestic experts.

\section{Neoadjuvant systemic therapy}

In the CSCO BC guideline, HER2 positive $\mathrm{BC}$ is one of the core factors in recommending neoadjuvant therapy. However, if HER2 positive is the only risk factor for a patient, our panelists agree that the diameter of the tumor lesion should be greater than $2 \mathrm{~cm}$ to avoid overtreatment.

Based on the NeoSphere trial (10), in 2015, the NCCN guidelines recommended an HP-containing scheme to treat early stage BC. However, it was not until 2020 that dual target therapy was included as a primary choice for HER2 positive patients in a neoadjuvant setting. The panelists agreed that dual target therapy is required if patients are suitable for neoadjuvant therapy. However, suspending the effective dual target therapy to receive a fluorouracil-epirubicin-cyclophosphamide (FEC) scheme after surgery is still controversial (11). More researches into extending THP courses to increase pCR rate and to avoid following an FEC regimen are needed. Comparatively, AC-THP became a favorable scheme over the THP-surgery-FEC strategy due to the continuous target therapy.

Platinum has always been an effective drug in a neoadjuvant setting. Since 2017, TCbH has been a level I recommendation in our guideline. This type of drug is irreplaceable even in the era of dual target therapy. The TRAIN-II study (12) has indicated that the addition of pertuzumab to $\mathrm{TCbH}$ would not significantly improve the cardiac toxicity of patients. The KRISTINE study (13) has also confirmed the effectiveness and safety of TCbHP in neoadjuvant therapy. On this basis, TCbHP has been included as a level I recommendation along with THP in our latest CSCO BC guideline. When choosing taxane, both docetaxel and paclitaxel are viable. Several clinical studies (14) have shown paclitaxel and nab-paclitaxel to have comparable survival benefit. Hence, nab-paclitaxel has become a more favorable option over paclitaxel.

\section{Adjuvant target therapy after neoadjuvant systemic therapy}

The KATHERINE trial (15), which compared adjuvant target therapy in patients who had residual invasive cancer at surgery after the completion of neoadjuvant therapy plus HER2-targeted therapy, demonstrated a superior diseasefree survival for those who received T-DM1 compared with those who received trastuzumab in an adjuvant setting. Given the rising importance of adjuvant target therapy after neoadjuvant therapy, we have added a new chapter offering recommendations based on different neoadjuvant antiHER2 target therapies and whether residual invasive cancer is present (Table 3). This is one of the most significant innovations compared with other influential global guidelines (Figure 3). 


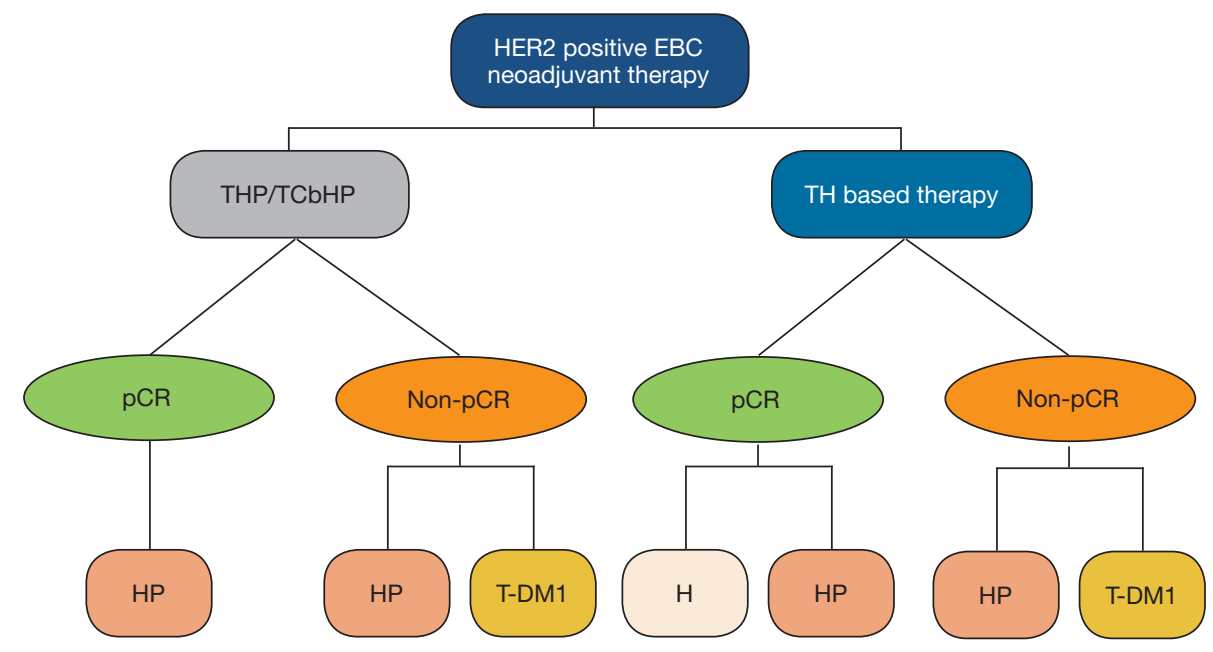

Figure 3 CSCO BC guideline strategies (version 2020): neoadjuvant and the following adjuvant target therapy. T, taxane; H, trastuzumab; $\mathrm{P}$, pertuzumab; T-DM1, ado-trastuzumab emtansine; CSCO, Chinese Society of Clinical Oncology; BC, breast cancer.

Patients who achieve pathologic complete response (pCR) after neoadjuvant therapy for HER2-positive $\mathrm{BC}$ are associated with substantially longer disease-free survival and OS (16). For those who have achieved pCR, there is strong evidence supporting the continuation of their original effective neoadjuvant targeted drugs during adjuvant therapy. However, for patients who received only trastuzumab during neoadjuvant therapy, a switch to trastuzumab-pertuzumab is deemed acceptable due to the probable benefit offered by pertuzumab.

Meanwhile, for those with residual invasive tumors, the CSCO BC guideline regard T-DM1 as a level I recommendation. Given the inaccessibility and poor cost effectiveness of T-DM1 in China, pertuzumab plus trastuzumab is also a reliable alternative. The continuous use of the original target drug is also acceptable, and is listed in the level II recommendations. However, we do not recommend the usage of trastuzumab alone as adjuvant therapy after poor response to neoadjuvant dual target therapy.

In summary, the CSCO BC guideline has developed rapidly over the past 4 years. Each update attests to the huge progress that is being made in cancer treatment, especially for HER2 positive BC. Pertuzumab, pyrotinib, and the recommendations for adjuvant therapy after neoadjuvant therapy represent the most important updates this year. From dual-targeted therapy to the post-trastuzumab era, there are more treatment options for HER2 positive patients. The CSCO BC guideline will continue to play an important role in promoting standardized treatment of BC and will further optimize targeted therapies in China and other countries.

\section{Acknowledgments}

Funding: None.

\section{Footnote}

Conflicts of Interest: Both authors have completed the ICMJE uniform disclosure form (available at http://dx.doi. org/10.21037/tbcr.2020.03.05). ZJ serves as an unpaid Editor-in-Chief of Translational Breast Cancer Research. JL serves as an unpaid Managing Editor of Translational Breast Cancer Research.

Ethical Statement: The authors are accountable for all aspects of the work in ensuring that questions related to the accuracy or integrity of any part of the work are appropriately investigated and resolved.

Open Access Statement: This is an Open Access article distributed in accordance with the Creative Commons Attribution-NonCommercial-NoDerivs 4.0 International License (CC BY-NC-ND 4.0), which permits the noncommercial replication and distribution of the article with the strict proviso that no changes or edits are made and the original work is properly cited (including links to both 
the formal publication through the relevant DOI and the license). See: https://creativecommons.org/licenses/by-nc$\mathrm{nd} / 4.0 /$.

\section{References}

1. Guidelines of chinese society of clinical onclogy breast cancer. Beijing: People's Medical Publishing House, 2019.

2. Swain SM, Baselga J, Kim SB, et al. Pertuzumab, trastuzumab, and docetaxel in HER2-positive metastatic breast cancer. N Engl J Med 2015;372:724-34.

3. Wardley AM, Pivot X, Morales-Vasquez F, et al. Randomized phase II trial of first-line trastuzumab plus docetaxel and capecitabine compared with trastuzumab plus docetaxel in HER2-positive metastatic breast cancer. J Clin Oncol 2010;28:976-83.

4. Perez EA, Lopez-Vega JM, Petit T, et al. Safety and efficacy of vinorelbine in combination with pertuzumab and trastuzumab for first-line treatment of patients with HER2-positive locally advanced or metastatic breast cancer: VELVET Cohort 1 final results. Breast Cancer Res 2016;18:126.

5. Ma F, Ouyang Q, Li W, et al. Pyrotinib or Lapatinib Combined With Capecitabine in HER2-Positive Metastatic Breast Cancer With Prior Taxanes, Anthracyclines, and/or Trastuzumab: A Randomized, Phase II Study. J Clin Oncol 2019;37:2610-9.

6. Jiang Z. Pyrotinib combined with capecitabine in women with HER2 + metastatic breast cancer previously treated with trastuzumab and taxanes: A randomized phase III study. Chicago: Proceedings of the ASCO Annual Meeting, May 31-June 4, 2019.

7. Cameron D, Piccart-Gebhart MJ, Gelber RD, et al. 11 years' follow-up of trastuzumab after adjuvant chemotherapy in HER2-positive early breast cancer: final analysis of the HERceptin Adjuvant (HERA) trial. Lancet 2017;389:1195-205.

8. Von Minckwitz G, Procter M, De Azambuja E, et al. Adjuvant Pertuzumab and Trastuzumab in Early HER2Positive Breast Cancer. N Engl J Med 2017;377:122-31.

doi: $10.21037 /$ tbcr.2020.03.05

Cite this article as: Li J, Jiang Z. CSCO BC guideline: updates for HER2 positive breast cancer in 2020. Transl Breast Cancer Res 2020;1:4.
9. Chan A, Delaloge S, Holmes FA, et al. Neratinib after trastuzumab-based adjuvant therapy in patients with HER2-positive breast cancer (ExteNET): a multicentre, randomised, double-blind, placebo-controlled, phase 3 trial. Lancet Oncol 2016;17:367-77.

10. Gianni L, Pienkowski T, Im YH, et al. 5-year analysis of neoadjuvant pertuzumab and trastuzumab in patients with locally advanced, inflammatory, or early-stage HER2-positive breast cancer (NeoSphere): a multicentre, open-label, phase 2 randomised trial. Lancet Oncol 2016;17:791-800.

11. Shao Z, Pang D, Yang H, et al. Efficacy, Safety, and Tolerability of Pertuzumab, Trastuzumab, and Docetaxel for Patients With Early or Locally Advanced ERBB2Positive Breast Cancer in Asia: The PEONY Phase 3 Randomized Clinical Trial. JAMA Oncol 2019. [Epub ahead of print].

12. Van Ramshorst MS, Van Werkhoven E, Honkoop AH, et al. Toxicity of dual HER2-blockade with pertuzumab added to anthracycline versus non-anthracycline containing chemotherapy as neoadjuvant treatment in HER2-positive breast cancer: The TRAIN-2 study. Breast 2016;29:153-9.

13. Hurvitz SA, Martin M, Symmans WF, et al. Neoadjuvant trastuzumab, pertuzumab, and chemotherapy versus trastuzumab emtansine plus pertuzumab in patients with HER2-positive breast cancer (KRISTINE): a randomised, open-label, multicentre, phase 3 trial. Lancet Oncol 2018;19:115-26.

14. Untch M, Jackisch C, Schneeweiss A, et al. NAB-Paclitaxel Improves Disease-Free Survival in Early Breast Cancer: GBG 69-GeparSepto. J Clin Oncol 2019;37:2226-34.

15. Von Minckwitz G, Huang CS, Mano MS, et al. Trastuzumab Emtansine for Residual Invasive HER2Positive Breast Cancer. N Engl J Med 2019;380:617-28.

16. Broglio KR, Quintana M, Foster M, et al. Association of Pathologic Complete Response to Neoadjuvant Therapy in HER2-Positive Breast Cancer With Long-Term Outcomes: A Meta-Analysis. JAMA Oncol 2016;2:751-60. 
Supplementary

Table S1 Updates in CSCO BC guideline from 2017 to 2020

\begin{tabular}{|c|c|c|c|c|}
\hline & 2017 & 2018 & 2019 & 2020 \\
\hline $\begin{array}{l}\text { Adjuvant therapy } \\
\text { after neoadjuvant }\end{array}$ & No recommendation & No recommendation & $\begin{array}{l}\text { Add the KATHERINE } \\
\text { trial in annotation }\end{array}$ & $\begin{array}{l}\text { The patients are recommended } \\
\text { according to neoadjuvant target } \\
\text { therapy and pCR status (detailed } \\
\text { in Table 2) }\end{array}$ \\
\hline $\begin{array}{l}\text { Adjuvant therapy } \\
\text { without prior } \\
\text { neoadjuvant (for } \\
\text { high risk patient) }\end{array}$ & $\begin{array}{l}\text { High risk patient }(T>2 \mathrm{~cm} \\
\text { or lymph node positive } \\
\text { or hormonal receptor } \\
\text { negative): } \mathrm{AC}-\mathrm{TH} \text { as basic; } \\
\mathrm{TCbH} \text { as optional }\end{array}$ & $\begin{array}{l}\text { High risk (lymph node positive } \\
\text { or } \mathrm{T}>2 \mathrm{~cm} \text { with other risks): } \\
\mathrm{AC}-\mathrm{TH} \text { or } \mathrm{TCbH} \text { as level I; } \\
\text { add dual-target therapy as } \\
\text { level II; add trastuzumab after } \\
\text { chemotherapy as level III }\end{array}$ & $\begin{array}{l}\text { High risk (lymph node } \\
\text { positive or } \mathrm{T}>2 \mathrm{~cm} \\
\text { with other risks): add } \\
\text { AC-THP as level II }\end{array}$ & $\begin{array}{l}\text { High risk (LN positive): add } \\
\text { TCbHP and AC-THP as level I; } \\
\text { AC-TH and TCbH downgrade to } \\
\text { level II; add neratinib as level III; } \\
\text { delete trastuzumab after } \\
\text { chemotherapy }\end{array}$ \\
\hline $\begin{array}{l}\text { Second line } \\
\text { therapy for MBC }\end{array}$ & $\begin{array}{l}\mathrm{LX}, \mathrm{H} \text { plus other } \\
\text { chemotherapy as basic; LH } \\
\text { and T-DM1 as optional }\end{array}$ & $\begin{array}{l}\text { LX as level I; H plus other } \\
\text { chemotherapy, T-DM1 as } \\
\text { level II; LH and L plus other } \\
\text { chemotherapy as level III }\end{array}$ & $\begin{array}{l}\text { Add pyrotinib plus } \\
\text { X as level II; H plus } \\
\text { other chemotherapy } \\
\text { downgrade to level III }\end{array}$ & $\begin{array}{l}\text { Add pyrotinib plus capecitabine } \\
\text { as level I; LX downgrade to level } \\
\text { II; add pyrotinib alone as level III; } \\
\text { delete H plus other chemotherapy }\end{array}$ \\
\hline
\end{tabular}

CSCO, Chinese Society of Clinical Oncology; BC, breast cancer; H, trastuzumab; P, pertuzumab; T-DM1, ado-trastuzumab emtansine; TKI, tyrosine kinase inhibitor; A, anthracycline; C, cyclophosphamide; N, vinorelbine; T, taxane; Cb, carboplatin; X, capecitabine. 